\title{
Symposium discussion: Paradigms, methodologies, and memory mechanisms
}

\author{
STANLEY FINGER \\ Washington University, St. Louis, Missouri
}

One of the advantages of being the moderator or discussant at a symposium is that one can indeed present some random thoughts and ideas about the particular topic. In this regard, one of the thoughts that keeps recurring in my mind is that after years and years of research we still know scant little about the neural mechanisms underlying learning and memory. This is certainly not due to lack of interest-the study of memory and how it may be mediated has always been a most attractive topic to individuals in the neural and behavioral sciences. In contrast, I think we must seriously question whether some of the paradigms being used to study memory are indeed appropriate, or whether they could be contributing to some of the confusion in this field. The issue is whether we are really measuring what we think we are measuring, or whether some of our approaches may only be capable of providing us with ambiguous results. I will argue that brain lesion and drug experiments can be useful tools for studying memory, but that the results of these studies can also be woefully misleading when not fully thought out.

The problem is one of interpretation. Unfortunately, we often are too quick to conclude that a "learning" deficit following a specific lesion is evidence that the structure destroyed represents a critical neural center, or at least a vital component part in an essential system. As von Monakow (1914) recognized around the turn of the century, it is not possible to damage one part of the brain without also affecting other partseven parts that may be some distance away. Thus, while the deficits following a lesion can be due to the primary effects of damaging a specific brain area, they can also be due to the secondary effects of diaschisis, metabolic changes, edema, or neuronal degeneration which may deprive another area of some of its inputs (Finger \& Stein, 1982; Schoenfeld \& Hamilton, 1977). Seen in this light, the inescapable conclusion is that a performance deficit cannot be

This contribution was presented as part of an invited symposium at the Midwestern Psychological Association meetings, Chicago, May 1984, entitled "Long-term memory: How durable, and how enduring?" The author would like to thank Donald Stein with whom many of these ideas were developed. The author's mailing address is: Department of Psychology, Washington University, St. Louis, MO 63130. taken as proof that the structure destroyed is in fact mediating the function being studied.

The experiments of Donald Meyer, Thomas LeVere, and their students and colleagues bear directly on this point. They examined visual memories and higher order reflexes (e.g., placing responses) that at first were thought to be lost after cortical lesions. These investigators were able to demonstrate that by changing task and demand characteristics, altering motivational conditions, or administering stimulant drugs, engrams for effectively executing these so-called "lost" programs still existed in the brain. The substrates for these memories were not destroyed in their entirety as had first been surmised. Obviously, the performance deficits seen in the earlier experiments were wrongly viewed as hard evidence that the structures destroyed were necessary for guiding the behaviors being studied.

It is also important to recognize that an area may well be playing an important role in some function like learning and memory in the intact brain, but that a deficit might not appear after a lesion in such an area if other areas are also doing some of the same things. In this case, one may erroneously conclude that the target area had little to do with the function being studied-a conclusion that might be at variance with the idea that memory is replicated throughout wide expanses of the brain. Such interpretative errors can easily be made if one does not realize that lesion effects do not necessarily reveal what a structure is doing in the intact brain. Nevertheless, lesion effects may allow us to begin to understand what the rest of the brain may be capable of doing following the loss of some of its parts, and this is important.

A logical problem very much related to these is a tendency to equate symptoms with functions, something John Hughlings Jackson (1879) warned against about 100 years ago. Jackson realized that although symptoms could be studied and measured after brain lesions, functions were constructs that could only be inferred from symptoms. Through the years, many analogies have been used to make the point that the jump from "symptoms" to "functions" is not easy, and that commonsense intuitions can be misleading. My favorite example of this involves the removal of a transistor from a radio and the resulting hissing and buzzing sounds. On the basis of these "symp- 
toms," can we really conclude that the function of the transistor was to serve as a hissing or buzzing "suppression center" in that radio? In 1910, Morton Prince, a past president of the American Neurological Association, tried to warn his colleagues about this type of thinking when he wrote:

The present doctrine of cerebral localization regarded as a mapping of the brain into areas within which lesions give rise to symptoms is one of the triumphs of neurology which cannot be valued too highly. ... [But] regarded as a localization of the psychophysiological functions represented by these symptoms within narrowly circumscribed areas, it is in large part naive to a degree which will excite the smiles of future neurologists (p. 340).

Thus, lesion data are not as easy to interpret as they are often made out to be. This is especially so in experiments on higher order functions, such as those attempting to understand memory for events, things, and places where deficits could be due to any of a number of factors, such as motivation, fatigue, emotion, and attentional dysfunctions, in addition to difficulties with memory storage and retrieval. But rather than single out the lesion study for criticism, it might be sobering to ponder the possibility that comparable problems also exist with other techniques that are currently being used to study the neural basis of learning and memory. Consider those experiments on the pharmacology of memory in which a drug is given to alter the levels of a neurotransmitter or the characteristics of a transmitter system. Conclusions about the functions of the system are often made on the basis of how one transmitter level correlates with scores on perceptual learning or memory tasks. But since there are many neurotransmitters in the brain, and because a balance exists among the different systems, a change in one system would have to affect the functioning of other systems. These secondary effects, or the resulting imbalance between transmitter systems, could be responsible for, or could underlie, the observed deficits.

I do not wish to convey the impression that drug studies and lesion experiments are of no value in studying memory. The point is only that, in terms of substrates, the results of these investigations must be regarded as possibilities or theories and not as facts about structure-function relationships. Even when many different methodologies are combined ("convergent operations"), the complex interrelationships among brain parts may still make it difficult, if not impossible, to talk definitively about the locus of "memory centers" in the brain as if they were easily localized compartments that could function independently of the remainder. Zangwill (1963) stated this clearly when he wrote:

There can be no doubt that a variety of behavior patterns may be evoked by stimulation, or lost after ablation, of limited parts of the brain. But I do not think we should therefore postulate special "centres," that is to say, assemblies of neurons whose function it is to generate the behaviour in question. As Sherrington clearly saw, behaviour does not spring ready-made from the brain, like Athene from the head of Zeus. Rather it must be regarded as an integration of a large number of component activities. . . . No one level, not even the highest, can properly be regarded as the exclusive seat of a behaviour pattern (pp. 338-339).

The difficulties involved in defining structurefunction relationships in the brain are not reasons to abandon these methodologies in the pursuit of understanding memory in healthy and damaged brains. However, as Donald Stein and I have suggested in our recent monograph on recovery from brain damage (Finger \& Stein, 1982), the questions that we might choose to ask with these methodologies need not be the same as those traditionally asked. For example, we have always been amazed at the variability that may follow brain lesions that appear quite comparable in different neurological patients. Although this variability in behavior is rarely addressed in our textbooks, the fact of the matter is that it is not uncommon to find one individual severely incapacitated by a lesion that may have only minimal effects on another person. One of the basic questions that could be asked concerns the factors that could account for such variability in symptomatology. Since one would be concerned with symptoms and not functions, the hypotheses generated by the experimenter would be directly testable. Furthermore, the outcomes of studies dealing with factors that can increase or decrease symptomatology might be meaningfully applied to how patients are treated in hospitals and clinics. Many of the experiments on CNS activation and motivation that were presented at this symposium can be viewed in this context and can be used to illustrate this point.

Research programs that proceed in this direction should be guided by the thought that paying attention to the lesion itself is not enough: the variability typically observed after specific brain lesions demands that one attend to the condition of the remaining brain as well as to the lesion. The analogy here may be to the doughnut and the hole, the lesion being the hole and the doughnut being the remaining brain. With regard to learning and memory, or any other function for that matter, the conclusion that one must inevitably come to is that the two must be treated as equal partners if we are to begin to understand the diversity of responses to brain lesions that look alike.

The fabric upon which the lesion is superimposed may be altered by previous learning and experience. This is shown by experiments on training and overtraining and also by studies on enriched and deprived environments (Finger \& Stein, 1982; Will \& Eclancher, 
1984). It can also be affected by drugs such as the amphetamines, phosphatitylcholine, and magnesium pemoline. Another important factor is how rapidly the lesion is growing. Tumors and strokes in the same areas, for example, typically have markedly different effects. Many examples of "serial lesion" effects have been presented by Donald Meyer, Thomas LeVere, and myself over the years (see Finger \& Stein, 1982), and the robustness of this phenomenon never ceases to amaze me. Furthermore, in my laboratory we have now found that early dietary conditions can also affect the response to focal brain lesions later in life (Finger \& Green, 1982; Mangold, Bell, Gruenthal, \& Finger, 1981). I am sure that there are many other factors, yet to be discovered, that can modify the backdrop upon which the lesion is superimposed. In fact, on a theoretical level, these experiments have led some investigators (e.g., Stein \& Firl, 1976) to propose that structure-function relationships are constantly changing in the brain.

Experiments on variables such as these can go far toward helping us to understand why there are individual differences in response to brain damage without bogging us down in blind alleys or unresolvable theoretical arguments over structure-function relationships as if each part and subpart of the brain were a known element carrying out its unique chores in a vacuum. By looking at both the lesion and the condition of the remaining brain, we should not be surprised by the wealth of new findings showing that under some conditions memories thought lost can be retrieved. In the latter case, the error was only in thinking that the deficits described previously were due to losses of essential substrates. Unfortunately, these questionable interpretations about the status of memory mechanisms may have effectively precluded more rigorous attempts to help certain patients with memory disorders. The papers presented at this symposium on the remarkable stability of memory certainly justify a more optimistic orientation. They may also justify a paradigm shift (Kuhn, 1977).

\section{REFERENCES}

Finger, S., \& Green, L. (1982). Early undernutrition and later hippocampal damage: 1. DRL performance in rats. Nutrition and Behavior, 1, 195-206.

Finger, S., \& STEIN, D. G. (1982). Brain damage and recovery: Research and clinical perspectives. New York: Academic Press.

JACKson, J. H. (1879). On affections of speech from disease of the brain. Brain, 2, 323-356.

Kunn, T. S. (1977). The essential tension. Chicago: University of Chicago Press.

Mangold, R. F., Bell, J., Gruenthal, M., \& Finger, S. (1981). Brain damage and undernutrition: A preliminary investigation. Brain Research, 230, 406-411.

Prince, M. (1910). Cerebral localization from the point of view of function and symptoms. Journal of Nervous and Mental Diseases, 37, 337-354.

Schoenfeld, T. A., \& Hamilton, L. W. (1977). Secondary brain changes following lesions: A new paradigm for lesion experimentation. Physiology \& Behavior, 18, 951-967.

Ste in, D. G., \& FirL, A. C. (1976). Brain damage and reorganization of function in old age. Experimental Neurology, 52, 157-167.

von Monakow, C. (1914). Die Localisation im Grosshirn under der Abbau der Funktion durch Kortikale Herde. Wiesbaden: Bergmann.

Will, B., \& ECLANCHER, F. (1984). Early brain damage and early environment. In S. Finger \& C. R. Almli (Eds.), Early brain damage: (Vol. 2). Neurobiology and behavior (pp. 349-367). New York: Academic Press.

ZANGWILL, O. L. (1963). The cerebral localisation of psychological function. Advancement of Science, 20, 335-344.

(Manuscript accepted for publication May 31, 1984.) 